\title{
Refractarios: un aspecto clave en la industria del cemento
}

PERCY CASTILLO NEIRA*, Ingeniero Químico AREQUIPA-PERU

\begin{abstract}
$R E S U M E N$
Para establecer un juicio de valor respecto al nivel téenico alcanzado en una planta cementera resulta sorprendentemente objetivo analizar el trabajo, planificación y control que se desarrolla alrededor del tema de Refractarios.

Considerarlo y reconocer la validez de este axioma pragmático ofrecerá inusitadas perspectivas de superación de indices de productividad y récords de producción.

En este trabajo se pretende un aporte a tal objetivo proporcionando algunas consideraciones

teórico-prácticas sobre las condiciones que se presentan en el sistema Horno Intercambiador-Enfriador.

más difundido, analizando las características que debe reunir el material refractario para cada zona $y$,

finalmente, incluyendo algunas apreciaciones

y recomendaciones principalmente de criterio, respecto

a algunos de los múltiples aspectos que forman parte

de uno de los campos más interesantes y complejos

de la industria del cemento.
\end{abstract}

\author{
$S U M M A R Y$
}

To sit in judgement values of the technic level reached in a cement plant, it is surprisingly objetive to analyse work, planning and control development about Refractories subject.

Considering and recognizing the validity of this pragmatic axiom it will offer unexpected improvement perspectives of the productivity rates and production records.

This work pretends to be a contribution for this purpose, bringing some theoretic and practical accounts about de conditions offered by the Kiln-Exchanger-Cooler most difussed system, analysing the characteristics that refactory material must have, in accordance with each zone, and finally, including some appraisals and recommendations regarding some of the manifold aspects which belongs to one of the most interesting ad complex fields of cement industry.

\section{INTRODUCCION}

El empleo de materiales refractarios en el proceso de clinkerización constituye, además de uno de los más fascinantes e interesantes problemas para el Ingeniero de Planta, un factor de importancia decisiva para el cumplimiento de los objetivos de la industria cementera: la producción de un cemento de óptima calidad al menor costo de fabricación posible.

En la operación del Horno Rotatorio únicamente puede anteponerse al rendimiento y la calidad del clínker producido, la seguridad de maquinaria y equipos; por tal razón, al existir condiciones de peligro de sobrecalentamiento de la chapa metálica, por problemas en el refractario o la costra que lo protege, se deben realizar maniobras operativas orientadas a superar la situación de peligro, pero que pueden afectar gravemente los récords de producción e, incluso, la calidad del clínker almacenado.

El trabajo que se desarrolle en este campo influirá poderosamente en los costos de producción, tanto por el costo directo de los materiales refractarios empleados, como por su participación dinámica durante la marcha del proceso.

\footnotetext{
* Consultor técnico para Iberoamérica en el tema de Ahorro de Energia en la Industria del Cemento.
} 
Su importancia técnica es de tal naturaleza, que los resultados que se logren pueden ser considerados como un parámetro muy objetivo de la calidad del trabajo que se desarrolla en una fábrica y, también, nivel técnico, capacidad y criterio de los profesionales que tienen a cargo su directa conducción.

Los factores que influyen en el trabajo de refractarios, en el proceso, son tan variados y complejos que requieren un planteamiento especifico individual para cada caso particular. En tal sentido resultaría equivocado y aun peligroso pretender planteamientos de validez general, y de gran importancia el criterio con el que se enfoque cada sistema individual, para lo cual resultará indispensable un profundo conocimiento de las características y variables que se presenten.

Entre el correcto cumplimiento de las sucesivas etapas del proceso y el adecuado funcionamiento del revestimiento refractario se produce una simbiosis de cuya realización depende el nivel de eficiencia del sistema.

La tecnología, modernos equipos y sistemas de fabricación permiten la disponibilidad de una abundante oferta en calidad y cantidad de materiales refractarios para la industria cementera; sin embargo, por las características tan particulares expuestas, se puede afirmar que la influencia de la calidad del refractario usado sólo influye en un $40 \%$, en la posibilidad de lograr mayores rendimientos y campañas más largas, dependiendo el $60 \%$ restante de factores ligados a su correcta elección, colocación y empleo.

Hacia tales aspectos se dirige este trabajo, fijando como objetivo central el lograr un aporte al criterio con el que debe enfocarse el tema, antes que pretender planteamientos exactos o detallados sobre el mismo.

\section{CONDICIONES DEL SISTEMA}

El avance tecnológico provocado por la crisis energética y la retracción de ventas consiguiente han determinado que las nuevas instalaciones proyecten su modernización y se orienten a los sistemas que permiten optimizar el consumo de energía $y$, por ende, menores costos de producción.

Se pueden considerar en términos generales:

$<200$ t clínker/dia: Hornos verticales.

$200-2,000$ t/día: Hornos con intercambiador de gases en suspensión.

$>2,000 \mathrm{t} /$ dia: $\quad$ Horno con intercambiador de gases en suspensión con sistema de pre-calcinación.

Aunque la tendencia a instalar unidades de mayor capacidad se justifica por las ventajas de espacio conseguidas con la instalación de cámaras de pre-calcinación, que permiten acortar sensiblemente la longitud del Horno, el sistema de Horno Vertical para pequeñas capacidades es una posibilidad que puede resultar conveniente para zonas con escasas vías de comunicación, limitados recursos financieros y disponibilidad local de carbón y/o otros combustibles sólidos. El diseño e instalación de refractario en los Hornos Verticales resulta mucho más simple en función a estar sometido a menores tensiones y exigencias, por lo cual no será objeto de estudio en este caso.

Otras opciones de elección, entre las cuales destaca el sistema Lepol, dependerán de características impuestas generalmente por las materias primas. 
En el sistema Horno - Intercambiador - Enfriador, con o sin cámara de Precalcinación, las condiciones a que será sometido el revestimiento refractario será de la mayor variedad y exigencia para cada zona, pero resulta de gran importancia considerar que, cada punto del sistema, requiere el máximo de atención, observación y control desde la toma de decisiones para el diseño original, su control y evaluación en trabajo y los posibles ajustes para el recambio o reparación.

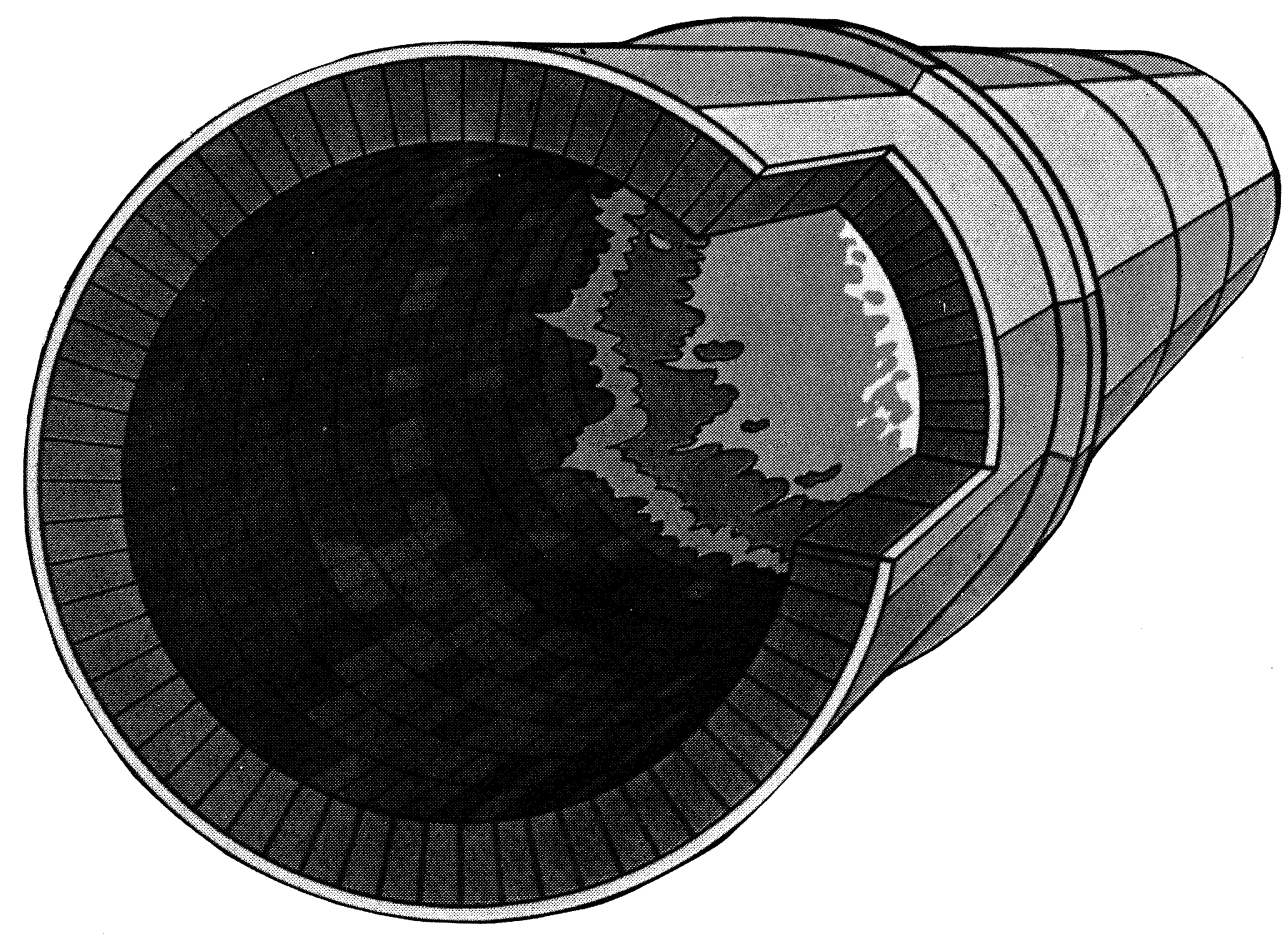

Fig. 1.-(Aristegui. Revista Cemento Hormigón, n. 619).

\subsection{En el Intercambiador}

El material ingresa en el ducto que asciende con gases hacia la primera atapa de cicloneo a unos $60^{\circ} \mathrm{C}$, pasando sucesivamente a través de las otras etapas y llegando al Horno, o la cámara de pre-calcinación, a unos $700-800^{\circ} \mathrm{C}$. Los gases ingresan provenientes del Horno a aproximadamente $1.000-1.100^{\circ} \mathrm{C}$, dejando el Intercambiador a $350-400^{\circ} \mathrm{C}$ luego de ceder su calor al material en los ductos ascendentes $(80 \%)$ y en los ciclones.

Dentro de estos rangos de temperatura se produce la exigencia térmica a que será sometido el refractario.

Las condiciones abrasivas del material revisten mayores exigencias, considerando la acción de las particulas arrastradas por los gases sobre las paredes de los tubos de ascensión, los ciclones y los tubos de descarga, a velocidades que pueden ser considerables. La abrasividad del polvo varia de acuerdo a su contenido de sílice y tamaño de las partículas, pero dentro de un estrecho margen, por lo cual puede ser cuantificado su efecto convenientemente.

El aspecto que quizás requiera mayor atención en esta etapa, por ser el que produce mayores daños y condiciones agresivas, es el de incrustaciones y ataque quimico, especialmente por la presencia de álcalis, cloruros y sulfatos en el crudo y el combustible. Estos materiales se volatilizan en el Horno y forman circuitos internos, concentrándose en las últimas etapas del 
Intercambiador, que crean condiciones propicias para que se produzcan incrustaciones, pegaduras y atoros de material, con gravísimas consecuencias para la continuiciad y normalidad del proceso.

Para evitar o corregir estos problemas se emplean medios manuales o neumáticos de limpieza, que dañan inmediata o gradualmente el revestimiento refractario.

Además de las medidas de carácter operativo que deben tomarse para evitar este efecto, desviando parcialmente gases hacia un bypass para disminuir niveles peligrosos de concentración, estableciendo un adecuado equilibrio de módulo de sulfatos para disminuir la volatilidad de álcalis o controlar la presencia de cloro necesario para elevarla, debe considerarse en el diseño de refractario las condiciones que faciliten la limpieza de incrustaciones sin daño del mismo.

\subsection{En la Cámara de Precalcinación}

La exigencia térmica resulta bastante fuerte debido a la presencia de quemadores de combustible que pueden alimentar aproximadamente el $60 \%$ del total; considerando que el crudo es descarbonatado en un $90 \%$ y esta reacción es fuertemente endotérmica, la transferencia de calor en el interior de la cámara es máxima. El fundamento mismo de la Precalcinación se basa en la máxima exposición de la superficie del crudo y el inmediato aprovechamiento del calor producido al combustionar, por parte de las partículas de polvo que deben encontrarse en los alrededores; cumpliéndose este efecto no existe peligro de sobrecalentamiento en las paredes del refractario, pero el mismo puede producirse por una deficiencia en el equilibrio perfecto que debe mantenerse.

Las condiciones referentes a rozamiento o abrasión son menos exigidas que en un ciclón de separacion, pero siempre considerables.

El riesgo de incrustaciones y pegaduras radica principalmente en la posibilidad de que se produzca una clinkerización incipiente, con presencia de fundidos por exceso de carga térmica, y de naturaleza más agresiva para el refractario.

Las instalaciones con el sistema de Precalcinación deberán considerar condiciones más exigentes desde el punto de vista térmico $\mathrm{y}$, consecuentemente, un cuadro diferente en lo referente a presencia de volátiles que deberá ser cuidadosamente planificada.

\subsection{En el Horno}

La diferencia entre las condiciones de trabajo del refractario en el Horno Rotatorio, respecto al Intercambiador y Enfriador, es bastante notable en razón de los esfuerzos mecánicos a que se somete durante el giro del Horno, lo cual debe ser tomado en cuenta en el diseño y colocado.

Pueden considerarse 4 zonas de caracteristicas bien definidas a lo largo del Horno Rotatorio, las cuales ofrecen diversas condiciones que debe soportar el refractario.

\subsubsection{Zona de Calentamiento o Descarbonatación}

En lo referente a exigencia térmica, aunque no se presentan aún temperaturas altas, la caracteristica de reacción fuertemente endotérmica de la descarbonatación exige la ubicación de ladrillo aislante.

La abrasión representa una exigencia mayor en el anillo de entrada de material y el ducto de gases, siendo considerable en toda la zona que no forma costra protectora. 
Pueden producirse problemas importantes debido a la formación de pegaduras y reacciones quimicas por la presencia de ciclos de volátiles, principalmente sulfatos y cloruros alcalinos.

\subsubsection{Zona de Transición y Nodulización}

Exige condiciones térmicas que pueden variar dentro de un espectro amplio, de acuerdo a las variaciones operativas del sistema; a diferencia de las zonas anteriores la temperatura, en la zona más alta, ya requiere una especial resistencia por parte del refractario, llegando a 1.300 $1.350^{\circ} \mathrm{C}$.

Al comenzar a formarse la fase líquida en esta zona, varían las condiciones fisicoquímicas del medio en que se desarrollan las reacciones del proceso y las que debe soportar el refractario.

Las características de la fase líquida formada, influenciada por la temperatura en aumento progresivo, resultan determinantes en la posibilidad de formación de una costra que puede proteger convenientemente el ladrillo en esta zona pero, con frecuencia, llega a formar los anillos que causan serios problemas operativos y, al derribarlos, dañan el refractario de la zona. La tensión superficial y la viscosidad de la fase líquida deben ser controladas de tal manera que la Nodulización se produzca adecuadamente y en el momento conveniente, lo que además de resultar decisivo para el proceso y la calidad del clínker producido, favorecerá la duración del refractario.

\subsubsection{Zona de Clinkerización}

Es la etapa culminante del proceso y, a su vez, la que resulta más exigente para el refractario. La temperatura puede alcanzar hasta $1.500^{\circ} \mathrm{C} \mathrm{y}$, a tales condiciones de exigencia térmica, se suman: la abrasión del clínker formado, la corrosión creada por la reactividad del material y las exigentes tensiones mecánicas creadas por la dilatación térmica de la chapa metálica, el refractario y la costra formada, la torsión creada por el giro del horno, la presión de los ladrillos producto de la pendiente del Horno, la fuerte variación de temperaturas de exposición a que se somete el ladrillo en cada revolución del horno, etc.

Se producen las reacciones de formación de las fases mineralógicas, que dan lugar a las caracteristicas del clínker formado.

Estas condiciones tan exigentes motivan que, para la protección del ladrillo refractario, presente la particularidad de constituir - por reacción con el clínker en formación - una costra protectora de cuya estabilidad dependerá, en gran parte, el rendimiento del refractario. El equilibrio entre corrosión e incrustaciones de la costra ya formada representa un complejo problema dependiente de muchos factores, cuyo conocimiento y control permitirá alcanzar los objetivos del proceso.

\subsubsection{Zona de Enfriamiento}

En la zona que transcurre el material, hasta su caida al enfriador, las condiciones térmicas para el refractario son bastante exigentes, disminuyendo gradualmente hacia la boca del Horno. Las condiciones varian de acuerdo a la posición del quemador respecto al anillo de salida. Las condiciones de abrasión, por parte del clínker formado, se hacen más exigentes en los últimos 2 ó 3 metros, porque generalmente no se forma costra. En esta zona pueden mencionarse las condiciones que se presentan en el cabezal o caperuza del Horno, y que debe soportar el refractario de protección.

Además de la temperatura elevada y variable, se debe mencionar la abrasión del polvo de clinker arrastrado por el aire secundario proveniente del enfriador. 


\subsection{En el Enfriador}

Aparentemente en el enfriador de parrilla no se presentan mayores exigencias para el material refractario, pero en muchos casos sucede todo lo contrario.

La exigencia térmica puede representar problemas únicamente en la zona de conexión con el Horno, donde también se produce abrasión por las partículas que arrastra el aire secundario.

La zona de caida de material del Horno es sometida a fuertes esfuerzos de impacto y abrasivos y, de formarse el "snowman" (hombre de nieve o árbol de navidad) por aglomeración del clinker, al removerlo se puede dañar gravemente el revestimiento refractario.

Las vibraciones y sacudidas que producen las parrillas en movimiento pueden afectar la estabilidad del refractario exigiendo, por ello, especial atención su colocado y diseño.

\section{CARACTERISTICAS Y PROPIEDADES REQUERIDAS EN EL REFRACTARIO}

Conocer las. principales caracteristicas de los materiales refractarios, que se utilizan o se podrian utilizar en la planta, resulta imprescindible para lograr un buen trabajo de la misma; planificar, prever y explicar el comportamiento del refractario proporciona un factor de control de grandes posibilidades para asegurar y mejorar rendimientos: en la contraparte, el desconocimiento de este campo provoca serios riesgos y pérdidas de producción al asumir causas equivocadas a los problemas que se presentan.

\subsection{Resistencia Mecánica en Frío}

Aunque este valor, normalmente expresado como Resistencia a la compresión en $\mathrm{kg} / \mathrm{cm}^{2}$, no puede asumirse para prever su exacto comportamiento en condiciones de operación, resulta de gran utilidad. como un punto de partida, para asumir en forma aproximada el mismo y, además, como factor de control de calidad que podria resultar indicativo de variaciones en las condiciones de fabricación. que provocarian cambios en su trabajo, en el momento de utilizarlos.

Se exige normalmente:

$350-500 \mathrm{~kg} / \mathrm{cm}^{2}$ para ladrillos de Alta alúmina (Acidos).

$500-700 \mathrm{~kg} / \mathrm{cm}^{2}$ para ladrillos de Dolomita o Magnesita (Básicos).

Para su determinación se emplea la Norma ASTM C-133.

\subsection{Poder Refractario}

Representa el comportamiento del material a altas temperaturas, y se expresa como una cifra con carácter abstracto deducida de un ensayo realizado en condiciones pre-establecidas. En Europa, se utiliza la medida de cono Seger y, en América, la medida correspondiente a la norma ASTM C-24 (Cono Pirométrico Equivalente). En la zona de clinkerización se emplea el cono Seger $\mathrm{N}^{\circ}$ 36. La estabilidad frente a la carga térmica bajo presión, se mide por la temperatura a la que el material refractario comience a reblandecerse bajo una presión de $2 \mathrm{~kg} / \mathrm{cm}^{2}$.

El refractario debe adoptar, a determinadas temperaturas, cierto grado de plasticidad; por ello es conveniente que este valor se ubique unos $150^{\circ} \mathrm{C}$ por encima de la temperatura de clinkerización. 


\subsection{Dilatación Térmica}

Tiene gran importancia conocer los coeficientes de dilatación de los refractarios y de los materiales que sean empleados con ellos en el colocado, asi como de la chapa metálica que se protege, a la temperatura de trabajo en cada caso. La alta temperatura que soporta la cara interior del ladrillo, en la zona critica, produce esfuerzos que provocan el "spalling" o desconchamiento de la cara superior; la resistencia del ladrilloal "spalling" se controla e incluso se halla normalizada (ASTM C-122).

Durante el diseño del colocado debe preverse la dilatación radial y longitudinal del ladrillo, existiendo todas las técnicas y elementos necesarios para lograr las condiciones que mejor se adapten a las de operación.

\subsection{Resistencia Química}

La agresividad del material, los gases o las cenizas del combustible se presentan en condiciones muy variadas, y el refractario en cada zona debe presentar la suficiente inercia química, o resistencia a ser atacado por las condiciones agresivas existentes.

La presencia de fase líquida favorece la acción agresiva de carácter químico, por lo cual este aspecto requiere especial atención en las zonas calientes del Horno.

En esta zona, sin embargo, resulta necesaria la formación de costra y, para ello, la parcial reacción del refractario con el material del proceso.

La investigación realizada por los fabricantes para adaptar el ladrillo a las exigentes condiciones requeridas permite ofrecer ladrillos de calidades muy convenientes, siendo de imprescindible importancia el contacto y asesoría técnica de los proveedores, para sacar el máximo provecho a los ladrillos adquiridos.

Para efecto de la resistencia a los volátiles agresivos, que se presentan, juegan importante papel la porosidad y permeabilidad de los materiales.

\subsection{Conductividad Térmica}

Las pérdidas de calor por radiación en el sistema representan aproximadamente el $10 \%$ del consumo en $\mathrm{kcal} / \mathrm{kg}$ de clínker, por lo cual la capacidad de aislamiento representa un permanente objetivo de control y mejoramiento mediante mejores diseños y sistemas.

El coeficiente de conductividad térmica $(\lambda)$ de los ladrillos silicoaluminosos, a $20^{\circ} \mathrm{C}$, es $1,00 \mathrm{kcal} / \mathrm{m} \cdot \mathrm{h} \cdot{ }^{\circ} \mathrm{C}$ y a $1.000^{\circ} \mathrm{C} 1,30 \mathrm{kcal} / \mathrm{m} \cdot \mathrm{h} \cdot{ }^{\circ} \mathrm{C}$. En el caso de los ladrillos de magnesita y dolomita (básicos) sucede al contrario: a $20^{\circ} \mathrm{C} 5,0 \mathrm{kcal} / \mathrm{m} \cdot \mathrm{h} \cdot{ }^{\circ} \mathrm{C}$ y a $1.000^{\circ} \mathrm{C} 3,0 \mathrm{kcal} / \mathrm{m} \cdot \mathrm{h} \cdot{ }^{\circ} \mathrm{C}$. La chapa del Horno tiene un $\lambda=40 \mathrm{kcal} / \mathrm{m} \cdot \mathrm{h} \cdot{ }^{\circ} \mathrm{C}$.

La conductividad y gradiente de temperatura al exterior, en conjunción con las caracteristicas fisicoquimicas del material y la temperatura en el interior del Horno, juegan un papel decisivo en la formación y conservación de la costra protectora.

Debe procurarse un equilibrio entre las condiciones óptimas para preservar la costra y evitar pérdidas de calor al exterior.

\subsection{Resistencia a la Abrasión}

Al igual que con los cementos, en el campo de materiales refractarios el criterio de calidad es 
relativo y el más apropiado para cada caso será el que mejor responda y se ajuste a las exigencias particulares requeridas. En los materiales que se emplean en el proceso de clinkerización, frecuentemente se comete el error de considerar sólo el factor temperatura y juzgar la calidad del refractario en función de su contenido en Alúmina. Considerando los ladrillos silicoaluminosos, la resistencia a la abrasión disminuirá con el incremento del porcentaje de Alúmina, y aumentará su resistencia a mayores temperaturas.

\title{
3.7. Porosidad y Permeabilidad
}

El tamaño, forma y cantidad de poros en los materiales refractarios revisten gran importancia en sus caracteristicas; con menor porosidad o mayor compacidad, se logra mayor resistencia al ataque quimico y a la abrasión, asi como más altas resistencias a compresión. La conductividad térmica también disminuye con la porosidad.

Una mayor porosidad influirá ventajosamente en la resistencia al "spalling" o "desconchamiento" ya referido.

La permeabilidad tiene gran importancia para la presencia de gases o fase liquida de caracteristicas muy agresivas.

\subsection{Resistencia al Choque Térmico}

En la operación del Horno, el objetivo ideal se orienta hacia la máxima conductividad en condiciones normalizadas pero, con frecuencia variable según el caso, se producen interrupciones

\author{
CUADRO 1
}

Tipos de refractarios y condiciones de trabajo por zonas en un Horno con Intercambiador de gases en suspensión y Enfriador tipo parrilla

\begin{tabular}{|c|c|c|c|c|c|c|c|c|}
\hline Partes del sistema & Refractarios necesarios & $\begin{array}{l}\text { Tempera- } \\
\text { tura máx. }\end{array}$ & $\begin{array}{c}\text { Formación } \\
\text { costra }\end{array}$ & $\begin{array}{c}\text { Desconcha- } \\
\text { miento }\end{array}$ & Abrasión & $\begin{array}{l}\text { Ladrillo } \\
\text { aislante }\end{array}$ & $\begin{array}{r}\text { Ataque } \\
\text { quimico }\end{array}$ & $\begin{array}{l}\text { Choque } \\
\text { térmico }\end{array}$ \\
\hline $\begin{array}{l}\text { Intercambiador (ci- } \\
\text { clones y ductos) }\end{array}$ & Silico-Aluminoso $30 \% \mathrm{Al}_{2} \mathrm{O}_{3}$ & $1.000^{\circ} \mathrm{C}$ & Indeseada & No & Exigente & $\mathrm{Si}$ & Escaso & Suave \\
\hline Cámara de enlace & Silico-Aluminoso $35 \% \mathrm{Al}_{2} \mathrm{O}_{3}$ & $1.200^{\circ} \mathrm{C}$ & Indeseada & No & $\begin{array}{l}\text { Muy exi- } \\
\text { gente }\end{array}$ & $\mathrm{Si}$ & $\begin{array}{l}\text { Conside- } \\
\text { rable }\end{array}$ & $\begin{array}{l}\text { Conside- } \\
\text { rable }\end{array}$ \\
\hline 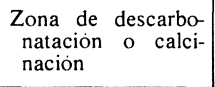 & Silico-Aluminoso $35-50 \% \mathrm{Al}_{2} \mathrm{O}_{3}$ & $1.250^{\circ} \mathrm{C}$ & Delgada & No & Poca & $\mathrm{Si}$ & Posible & $\begin{array}{l}\text { Conside- } \\
\text { rable }\end{array}$ \\
\hline $\begin{array}{l}\text { Zona de Transición } \\
\text { o Nodulización }\end{array}$ & $\begin{array}{l}\text { Silico-Aluminoso y Alta Alúmina } \\
\left(50-75 \% \mathrm{Al}_{2} \mathrm{O}_{3}\right) \text { Cromo-Mag- } \\
\text { nesita }(50-60 \% \text { de } \mathrm{MgO})\end{array}$ & $1.350^{\circ} \mathrm{C}$ & Probable & $\mathrm{Si}$ & Muy poca & No & Probable & Exigente \\
\hline $\begin{array}{l}\text { Zona de Enfria- } \\
\text { miento }\end{array}$ & $\begin{array}{l}\text { Cromo-Magnesita } 60 \% \mathrm{Mg} \text { y Alta } \\
\text { Alúmina }\left(70 \% \mathrm{Al}_{2} \mathrm{O}_{3}\right)\end{array}$ & $1.350^{\circ} \mathrm{C}$ & Necesaria & $\mathrm{Si}$ & $\begin{array}{l}\text { Muy Exi- } \\
\text { gente }\end{array}$ & No & Exigente & $\begin{array}{l}\text { Muy exi- } \\
\text { gente }\end{array}$ \\
\hline Anillo de salida & $\begin{array}{l}\text { Ladrillos extra-duros aluminosos } \\
\left(50-70 \% \mathrm{Al}_{2} \mathrm{O}_{3}\right)\end{array}$ & $1.250^{\circ} \mathrm{C}$ & Escasa & $\mathrm{Si}$ & $\begin{array}{l}\text { Muy exi- } \\
\text { gente }\end{array}$ & No & Escaso & Exigente \\
\hline Cabezal del Horno & $\begin{array}{l}\text { Ladrillos, castables o plásticos re- } \\
\text { fractarios }\left(50 \% \mathrm{Al}_{2} \mathrm{O}_{3}\right)\end{array}$ & $1.200^{\circ} \mathrm{C}$ & No & $\mathrm{Si}$ & Exigente & $\mathrm{Si}$ & Escaso & Exigente \\
\hline $\begin{array}{l}\text { Caida de material al } \\
\text { Enfriador }\end{array}$ & Resistentes al impacto $50 \% \mathrm{Al}_{2} \mathrm{O}_{3}$ & $1.200^{\circ} \mathrm{C}$ & $\begin{array}{l}\text { Aglomera- } \\
\text { ción }\end{array}$ & $\mathrm{Si}$ & $\begin{array}{l}\text { Muy exi- } \\
\text { gente }\end{array}$ & $\mathrm{Si}$ & Escaso & Probable \\
\hline Enfriador & Silico-Aluminoso $\left(50-30 \% \mathrm{Al}_{2} \mathrm{O}_{3}\right)$ & $\begin{array}{r}1.100- \\
400^{\circ} \mathrm{C}\end{array}$ & No & No & Exigente & $\mathrm{Si}$ & Raro & Suave \\
\hline
\end{tabular}


o enfriamientos que provocan variaciones bruscas de temperaturas afectando sensiblemente la vida del refractario. La resistencia del mismo a estos choques térmicos guarda estrecha relación con la porosidad del material y podria obtenerse, aumentando ésta, grandes resultados, pero se afectarian otras características igualmente importantes.

\section{DISEÑO Y COLOCADO ORIGINALES}

El costo directo de los materiales refractarios representan aproximadamente el $1 \%$ del costo total de inversión en una planta nueva, lo cual quizás sea la causa de un error fatal que se comete con frecuencia: no realizar el diseño y colocado del material refractario, adjudicándole la verdadera dimensión de importancia que tendrá en el futuro funcionamiento del sistema, y que resulta de carácter fundamental en el rendimiento de la planta y en los costos de producción.

Un trabajo que, sin llegar a ser deficiente, simplemente no cumpla los requerimientos mínimos de calidad que resultan indispensables en este campo, condenará al sistema de por vida a trabajar por debajo de su posible capacidad máxima, al tener que funcionar compensando deficiencias que, a su vez, crearán otras nuevas en un círculo interminable.

Un adecuado trabajo posterior del equipo técnico de planta puede conseguir resultados aceptables en la medida y gravedad que tales deficiencias hayan sido cometidas. Sin embargo, un trabajo defectuoso producirá resultados desastrosos y lamentablemente irreversibles, por la gravedad de sus consecuencias; tales casos representarán plantas con constantes paradas por problemas de caida de refractarios, campañas cortas por desgaste prematuro del ladrillo en la zona critica, altos consumos energéticos, problemas de anillos, atoros en los ciclones, etc.

En términos generales, algunos aspectos que deben considerarse para nuevas instalaciones son los siguientes:

- La planificación, diseño y colocado del refractario deber ser realizado por un equipo de especialistas y con participación del personal que tendrá a su cargo la operación de la planta.

- En base al conocimiento detallado del diseño de equipos y maquinaria, el Know-How del sistema, caracteristicas del material de alimentación y toda la información que resulte útil a este efecto, deben fijarse en las condiciones de marcha del sistema.

- Se fijarán todas las condiciones a las cuales estará sometido el material refractario en cada punto del sistema, estableciendo las zonas de requerimientos similares.

- Para cada zona se definirá el tipo de refractario más conveniente y las exigencias a que se verá sometido en las condiciones de operación normal y posibles exigencias no rutinarias, pero probables.

- Se elegirán los materiales refractarios con un criterio exclusivamente técnico tomando, en consideración que 1 metro lineal del Horno de revestimiento refractario equivale aproximadamente al costo de 8 horas de consumo de combustible; el considerar economias en la selección de refractarios bajo tales circunstancias resulta ridiculo y equivocado, si ello se contrapone a razonamientos técnicos.

- El diseño del colocado debe planificarse minuciosamente, tomando en cuenta toda la información acumulada referente a las condiciones dinámicas de trabajo, las exigencias de la puesta en marcha, los medios que generalizan su duración y estabilidad, y las caracteristicas de los materiales empleados que influyen en las tensiones a que será sometido el refractario y que deberá asimilar. 
Este diseño debe incluir la ubicación de juntas de dilatación, anclajes de fijación, materiales aislantes, condiciones de materiales vaciados o gunitados, herramientas necesarias, etc.

- El colocado de los materiales refractarios deberá ajustarse al diseño planificado minuciosamente, corrigiendo deficiencias o variaciones de las instalaciones con el mismo criterio y conocimiento utilizado durante el diseño.

- Anteponer una exigencia de tiempo a la calidad del colocado es la máxima torpeza que puede concebirse en el trabajo de planta.

- Considerando que son tan variados y complejos los factores que deben controlarse para el adecuado trabajo del refractario, y que el rendimiento teórico máximo posible depende de todos y cada uno de ellos, el diseño y ejecución del colocado son de vital importancia y trascendencia.

- El primer calentamiento y arranque del sistema deberá ser objeto del más cuidadoso y paciente planeamiento para evitar daños irreversibles en todo el sistema.

\section{CONTROL DE FUNCIONAMIENTO Y REPARACIONES}

"El sistema del Horno debe paralizarse únicamente para reparación de refractario". Este enunciado que define el $100 \%$ de continuidad de marcha, parámetro de gran interés e influencia en la productividad y el éxito económico de la empresa, resulta muy objetivo para mostrar la importancia que reviste un adecuado rendimiento del material refractario.

La influencia decisiva manifestada respecto a la planificación del diseño y colocado originales se prolonga, al igual que los objetivos perseguidos, en un permanente y adecuado seguimiento y control del trabajo del refractario en las condiciones de operación. El mismo jugará un papel decisivo en el alargamiento de la campaña del Horno y facilitará el control de las condiciones de operación que, correspondientemente, influenciarán la duración del refractario.

\subsection{Un control efectivo deberá considerar los siguientes puntos:}

- Desde la puesta en marcha del sistema se debe efectuar un control periódico, o permanente según lo requerido, en la temperatura exterior de todas las zonas revestidas de refractario; ello servirá como importante contribución al control del proceso para prevenir daños, en el refractario interior, que cause deterioros de mayor gravedad en la chapa del Horno.

- Se deberán prevenir y controlar las causas que provoquen esfuerzos exagerados para el revestimiento de todas las zonas, que lo dañen o debiliten. Algunas podrian ser:

* Control de formación, longitud y ubicación de la llama y las condiciones de combustión.

* Presencia por encima de límites permisibles, de volátiles o constituyentes peligrosos y agresivos para el refractario.

* Evitar los cambios bruscos de temperatura y prevenir sus efectos y consecuencias.

* Excesivas cargas térmicas provocadas por insuficiencia de alimentación o descontroles operativos.

* Evitar atmósferas reductoras asegurando el suficiente oxígeno para la combustión.

* Evitar la formación en el Horno y caída al enfriador de bolas y costras en el clínker. 
- La zona de clinkerización es la que debe determinar las condiciones para la paralización del sistema, reparación $\mathrm{y}$, por tanto, la formación de una costra estable de caracteristicas adecuadas es objetivo de gran interés. Para ello tendrán gran influencia:

* Carácterísticas fisico-quimicas adecuadas del crudo y máxima homogeneidad de las mismas.

* Establecimiento de una curva térmica ajustada a las condiciones más convenientes de cumplimiento de las diversas etapas del proceso.

* Gradiente de temperatura a través de la costra, ladrillo y chapa metálica que se mantenga uniforme en valores que resulten convenientes.

* La correcta y oportuna nodulización, favorecerá las condiciones que se presentarán en la zona de clinkerización.

* La forma de la llama, una vez normalizada en las condiciones más favorables, deberá ser mantenida estable.

* La temperatura del aire secundario deberá mantenerse estable.

- Los esfuerzos mecánicos a que es sometido el ladrillo en el interior del Horno son de gran influencia en su rendimiento, y deben merecer el máximo de atención y control:

* El giro del Horno, el peso de los propios ladrillos y la dilatación térmica de los mismos crean esfuerzos combinados de compresión, torsión, esfuerzo al corte, etc. que exigen ser compensados en el colocado, y que en operación requieren el máximo de normalización en las condiciones de marcha.

* Las pistas de apoyo que permanecen flotantes, manteniendo un desplazamiento respecto a las revoluciones del tubo del horno por calentamiento de este último, pueden llegar a presionarlo al dilatarse, deformando exageradamente su ovalidad, ya existiendo la misma por el peso del Horno en caliente. Esta situación es peligrosísima y bastaría que se produzca en alguna oportunidad para disminuir sensiblemente la duración del refractario, y por supuesto, de resultar exagerada o no controlarse, podria causar daños irreparables en la chapa del Horno.

* El desplazamiento longitudinal del Horno debe controlarse estrictamente, pues de lo contrario creará una variación de esfuerzos tensionales en el ladrillo que influirá en su rápida erosión.

- Como se ha mencionado, el máximo récord de continuidad de marcha (100\%), se refiere a que no existan paradas adicionales a las de reparación de revestimiento refractario de la zona crítica, ya sean por razones operativas o de mantenimiento. Tal objetivo exige que se cumplan requerimientos ajenos al refractario y resulta imprescindible considerar a este efecto de carácter vital, el Mantenimiento Preventivo.

5.2. La siguiente Reparación deberá comenzar a planificarse exactamente en el momento que concluye la anterior, es decir, cuando se pone en operación el sistema a través de la etapa de calentamiento.

Las actividades que se realizan cuando el sistema se halla paralizado por Reparación, tienen igual o mayor importancia que la operación misma en los récords de producción que se logren.

Es muy posible que la actual crisis de ventas de cemento, que se presenta hace algunos años y 
que provoca que sean raros los casos de fábricas que requieren el máximo de su capacidad instalada haya distendido notablemente la antigua urgencia de acortar al máximo los días de reparación, para lo cual se toma mayor tiempo y tranquilidad. Sin embargo, esta situación debe ser aprovechada para comprobar que la reparación siempre debe ser efectuada con el máximo de eficiencia y la planificación y control adecuados, nunca sacrificando calidad por tiempo. Las condiciones exigidas volverán y la ocasión debe ser aprovechada preparándose para ello.

Algunos de los aspectos que deben considerarse para una reparación de refractarios:

- Evaluación de los consumos de ladrillos en todas y cada una de las zonas mediante sondeos, mediciones e inspecciones que permitan elaborar minuciosamente el estado y condiciones de las zonas que no serán removidas, y tomar decisiones respecto a las zonas que sí lo requieran.

- Efectuar los trabajos de remoción, teniendo exigente cuidado de no afectar al resto de ladrillo. Normalmente los daños que se ocasionan en esta forma no son apreciables, pero sus consecuencias lamentables.

- Efectuar una minuciosa planificación y diseño de colocado, utilizando la tecnología necesaria para poner en práctica el criterio básico de que las instalaciones deberán ser realizadas teniendo como objetivo su trabajo en las condiciones de operación.

- El Horno debe girarse lo mínimo, considerando que la dilatación del ladrillo no es reversible sólo en un $30 \% \mathrm{y}$, por lo tanto, al estar frío queda parcialmente suelto.

- La colocación de ladrillo debe ser realizada con el máximo de dedicación y minuciosidad; bastaría un punto débil para afectar el buen trabajo realizado en todo el resto de la instalación.

- El personal encargado del colocado debe ser especializado en el tema.

Resulta plenamente justificado, para cualquier Empresa cementera, poseer su propio equipo de especialistas (sólo colocadores), que durante la época de operación puedan realizar tareas relacionadas con el sistema o formar parte del equipo de planta y servicios.

5.3. La garantía de los fabricantes de materiales refractarios basando sus apreciaciones en su excelente calidad son valederas, pero en un $40 \%$, que es la valoración de la influencia de la calidad de fabricación; el $60 \%$ restante dependerá exclusivamente de los factores muy parcialmente comentados en este artículo y otros de exclusiva responsabilidad del equipo de profesionales y técnicos de cada fábrica individual.

Finalmente, una aseveración que quizás preocupe a muchos Directores de Fábrica: La Tecnología actual, en este campo, permite asegurar que no deben producirse campañas de refractario inferiores a 1 año en una fábrica de cemento de caracteristicas normales de vía seca, en la cual se conduzca el tema de refractarios en forma adecuada y se reconozca su importancia, en su verdadera dimensión.

\section{Nota Bibliográfica}

El desarrollo del trabajo ha sido realizado combinando aspectos prácticos de experiencia directa del autor con la consulta bibliográfica del abundante material conocido sobre el tema y la gran variedad de artículos técnicos publicados. Hubiera resultado demasiado extenso incluir una relación de todo el material directa o indirectamente utilizado. Sin embargo, resulta importante 
mencionar el excelente trabajo "Materiales refractarios para la industria del cemento", del Dr. Francisco Soria Santamaría, Prof. de Investigación del IETcc, el cual, pese a haber transcurrido 30 años desde su publicación, es de gran valor y utilidad.

\section{publicaciones del i.e.t.c.c.}

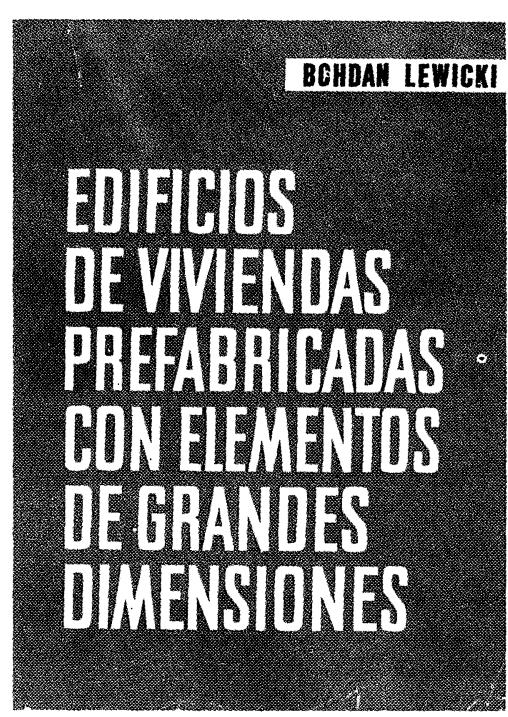

Bohdan Lewick

Este libro trata de los problemas relativos a la construcción de los edificios de viviendas o publicos realizados con elementos prefabricados de grandes dimensiones. Se han bricados de grandes dimensiones. Se han estudiado los problemas de arriostramiento,
asi como los que plantea la resistencia de asi como los que plantea la resistencia de
los elementos y de la estructura; se han examinado las cuestiones de orden higrotérmico, acústico y de resistencia al fuego: también se ha profundizado en el estudio de la estanquidad de los muros exteriores y de las juntas.

La obra incluye numerosas llustraciones que dan detalles de diversas soluciones, as como ejemplos de cálculo, tablas de valores numéricos, diagramas y ábacos.

Un volumen encuadernado en tela, de $24 \times 17 \mathrm{~cm}$, compuesto de 616 págs.

Precios: 2.500 ptas.; \$USA 36.00 .

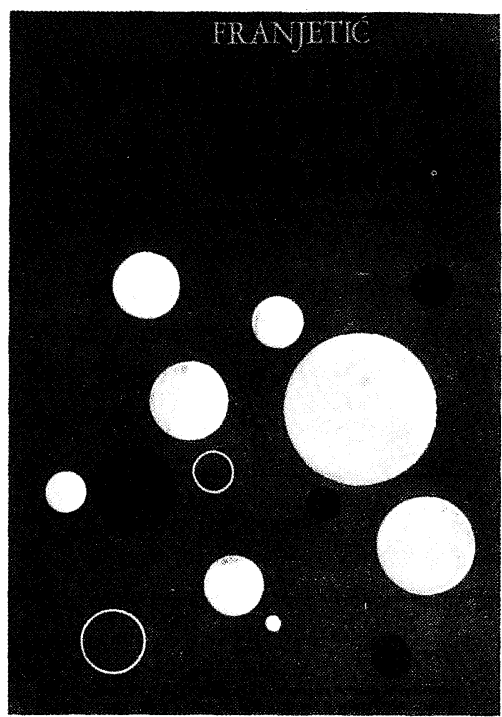

Zorislav Franjetic

En la obra de Franjetić se expone de una forma minuciosa, ordenada y sistemática todo un cuerpo de doctrina que reúne el conocimiento actual sobre el endurecimiento nocimiento actual sobre el endurecimiento
rápido del hormigón. Parte el autor de los principios básicos y llega a las últimas consecuencias y realidades técnicas y econo micas.

Es una obra de consulta, tanto para el investigador sobre la materia, como para el proyectista y el realizador y montador de plantas e instalaciones y equipos de curado y endurecimiento rápido.

Un volumen encuadernado en cartóné, de $17 \times 24,5 \mathrm{~cm}$, compuesto de 385 págs. 110 figuras y 10 tablas.

Precios: 2.500 ptas.; \$USA 36.00 .

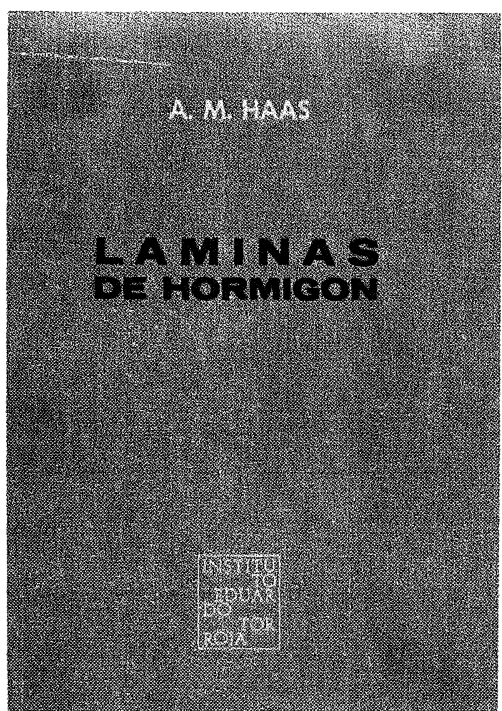

\section{A. M. Haas}

Al escribir este libro el autor intento poner a disposición de los estudiantes y de los ingenieros unos conocimientos prácticos, adecuados para servir de guia en el diseño y construcción de láminas delgadas de hormigón.

El autor está convencido de que el éxito en el diseño de una lámina exige, por parte del proyectista, un examen de las tres fases por las que pasa la materialización de la lámina: el diseño, el análisis estructural y la construcción de la estructura.

Un volumen encuadernado en tela, de $17 \times 24,5 \mathrm{~cm}$, compuesto de 420 págs., 141 figuras, 22 fotografias y 6 tablas.

Precios: 2.500 ptas.; \$USA 36.00 